\section{Heroingestützte Suchtbehandlung - Zwei Fallbeispiele}

Zusammenfassung: Die Poliklinik Zokl2 wurde 1993 im Rahmen des Forschungsprojekts zur ärztlich kontrollierten Verschreibung von Betäubungsmitteln (PROVE) aufgebaut. Heute bietet es 120 Plätze für heroingestützte Behandlungen an, wobei $2 / 3$ der Patient(inn)en Frauen sind, um eine geschlechtergerechte Behandlung sicherzustellen. Das Team besteht aus rund 30 Mitarbeiter(inne)n. Verschrieben wird vorwiegend Heroin in flüssiger oder oraler Form. Die Poliklinik ist im Zentrum Zürichs gelegen.

Das Fallbeispiel Iris B. beschreibt den langen Weg zur Abstinenz und zeigt, dass bis dahin eine Vielzahl von Zwischenzielen zu erreichen sind, wozu psychische und physische Stabilität sowie ein adäquates soziales Umfeld zählen. Mario K. ist ein Beispiel dafür, dass manche Patient(inn)en ihre Lebenssituation zwar zeitweise stabilisieren können, sofern sie eine konstante Begleitung erhalten, dass sie aber immer wieder schwere Krisenphasen erleben. Die Behandlung verfolgt langfristig das Ziel, die Lebenssituation zu stabilisieren.

Schlüsselwörter: Betäubungsmittel - heroingestützte Suchtbehandlung - Fallbeispiele

Zokl2 - Heroin-Supported Addiction Treatment: The Zokl2 polyclinic was set up in 1993 as part of the PROVE research project on the medically-supervised prescription of narcotics. Today, it offers 120 places for heroin-supported treatment, with 2/3 of the patients being women, so as to ensure the correct gender balance in the treatment. The team is made up of around $\mathbf{3 0}$ employees. Heroin in liquid and oral form is the chief narcotic prescribed. The polyclinic is located in the centre of Zurich.

The case study of Iris B. describes the long road to abstinence and shows that there are many intermediate targets to be met en route, including mental and physical stability and a suitable social environment. Mario $\mathrm{K}$. is one of those patients who can stabilise their situation in life from time to time, providing they are constantly accompanied, but who repeatedly experience severe crisis phases. Over a prolonged period, the treatment is aimed at stabilising the patients' situation in life.

Key words: Narcotics - Heroin-Assisted Treatment of Addiction - Case Studies

Suchttherapie 2000; 1: 95-97

(c) Georg Thieme Verlag Stuttgart · New York

ISSN 1439-9903
Anna-Barbara Villiger, Barbara Baier

ARUD Zürich, Poliklinik Zokl2

\section{Iris B.: Der lange Weg zur Abstinenz}

Die heute 38-jährige Iris B. ${ }^{*}$ meldete sich erstmals telefonisch Anfang April 1994. Sie ist seit 1984 heroinabhängig und hat schon zahlreiche ambulante Entzüge hinter sich, die leider immer wieder scheiterten. Sie begab sich deshalb 1989 in psychotherapeutische Behandlung, brach diese aber aus finanziellen Gründen ab. Gleichzeitig beendete sie die parallel dazu begonnene, ebenfalls rund fünf Jahre dauernde Methadonbehandlung, in der Hoffnung, gänzlich drogenfrei leben zu können. Nach sehr kurzer Zeit wurde sie aber rückfällig. Danach war Iris B. nicht mehr für einen Entzug zu motivieren, da ihr ein drogenfreies Leben als Illusion erschien. Einen Wiedereinstieg ins Methadonprogramm empfand sie als Rückschritt. Durch den Eintritt ins Programm für heroingestützte Behandlungen hoffte Iris B., ihr Drogenproblem längerfristig angehen zu können.

Iris B. ist in ländlicher Umgebung als Zweites von drei Kindern aufgewachsen. Der Vater hatte sich, aus einem technischen Beruf kommend, in einer Bank hochgearbeitet; die Mutter, ursprünglich Damenschneiderin, arbeitete von Zeit zu Zeit im Verkauf. Den Vater erlebte Iris B. als unberechenbar und gewalttätig. Der ältere Bruder wurde von ihm oft „drangenommen“, die kleine Schwester war sein Lieblingskind, Iris B. fiel die Mittlerrolle zu - sie versuchte, sich einerseits anzupassen, damit ihr nicht dasselbe widerfuhr wie dem Bruder, andererseits wurde sie von der Mutter schon als kleines Kind in deren Sorgen und Probleme eingeweiht. Die Mutter, die sie vor Gehässigkeiten und Wutausbrüchen des Vaters zu schützen suchte, war oft krank und musste mehrmals wegen schwerer Depressionen hospitalisiert werden. Iris B. galt als fleißiges, „problemloses“ Kind und absolvierte sowohl Schule als auch Ausbildung ohne Probleme. Nach Abschluss der Lehre arbeitete sie ohne Unterbrechung in verschiedenen Banken. Mit zweiundzwanzig kam sie mit harten Drogen in Berührung, nachdem sie schon seit Jahren Alkohol und Cannabis in mäßigen Mengen konsumiert hatte. Zunächst schnupfte sie nur gelegentlich Kokain, seltener auch Heroin; mit vierundzwanzig war sie dann opiatabhängig und 1994 wurde sie aus der Bank entlassen, da sie sich einer Vorgesetzten anvertraut und ihr den Drogenkonsum gestanden hatte. Beim Eintritt in die heroingestützte Behandlung war Iris $\mathrm{B}$. arbeitslos, hatte etwa fünfzigtausend Franken Schulden und verkehrte täglich in der Drogenszene.

Im Rahmen der Eintrittsgespräche wurde der allgemeine $\mathrm{Zu}$ stand der 32-Jährigen als leicht reduziert beschrieben. Im Gespräch war die Beziehung gut herzustellen, Iris B. wirkte auf- 
merksam und differenziert, erzählte ruhig und zusammenhängend. Auffällig war die in bestimmten Zusammenhängen plötzlich auftretende enorme Wut, die angesichts ihrer sonst so ruhigen Art überraschte. Ihren letzten „Absturz“ brachte sie in Zusammenhang mit der Beziehung zu ihrem Freund, der ihr „auf der Kappe gelegen“ und sie für seine Zwecke ausgenützt habe. Sie ziehe immer Männer an, die unselbständig seien und sich an starke Frauen klammerten, erzählte sie. Seinetwegen habe sie auch Schulden gemacht.

Iris B. wurde im April 1994 ins Programm aufgenommen und konsumierte dreimal täglich die Maximaldosis, das heißt total 21 Sugaretten (Heroinzigaretten). Zwischen April und Dezember 1994 besuchte sie Computerkurse, konnte im Rahmen eines Integrationsprogramms kurze Arbeitseinsätze absolvieren und bewarb sich parallel um Stellen. 1995 arbeitete Iris B. an insgesamt vier Temporärstellen, jeweils 100\%. Anfang 1996 wurde ihr eine Stelle bei einer Bank angeboten, die nach drei Monaten in eine feste Anstellung umgewandelt wurde. Kurz vor Stellenantritt entschloss sie sich zu einem Schnellentzug in Mailand. Anschließend wurde sie mit Nemexin behandelt. Im Dezember meldete sie sich wieder bei uns, hatte Hepatitis mit massiv erhöhten Leberwerten und musste schon einen Monat nach dem Entzug das Nemexin absetzen. Anschließend wurde sie wieder rückfällig, schnupfte Heroin und Kokain. Sie begann bei ihrem Arzt ein Methadonprogramm, wurde wiederholt rückfällig - eine Wiederaufnahme ins Heroinprogramm war angezeigt.

Nach dem Wiedereintritt im Dezember 1996 sowie nach Umstellung auf Herointabletten im Februar 1997 zeichnete sich eine stetige Stabilisierung ab. Iris B. hatte sich mit ihrem Anspruch, um jeden Preis abstinent leben zu müssen, immer wieder überfordert, jetzt konnte sie sich darauf einstellen, dass sie noch für eine gewisse Zeit heroinabhängig bleiben würde. Diese Zeit nutzte sie, um einige große anstehende Probleme zu lösen. So hat sie innerhalb der letzten vier Jahre Fr. 50000,- Schulden abgezahlt, die Interferon-Therapie sehr diszipliniert und bis jetzt erfolgreich begonnen und wieder einen Bekanntenkreis außerhalb der Drogenszene aufgebaut. Seit zwei Jahren bewohnt sie gemeinsam mit ihren Eltern und ihrer Schwester ein großes Haus. Sie hat ihre Position in der Bank verbessern können und sich dabei gegen ehrgeizige Konkurrenten durchgesetzt.

Das Ziel, abstinent zu leben, hat sie nicht aufgegeben. Sie hat in den letzten vier Jahren ihre Lebensumstände so verändert, dass es nun ein realistischeres Ziel ist.

\section{Mario K.: Zokl2 - ein Halt in schwieriger Zeit}

Mario K.s* Eltern stammen aus bäuerlichen Verhältnissen in Süditalien. Er selber kam 1965 in der Schweiz zur Welt. Sein Vater machte hier Karriere in einer Baufirma, die Mutter war Hausfrau. $\mathrm{Zu}$ ihr hatte er engeren Kontakt als zum Vater. Die Eltern und seine jüngere Schwester zogen 1993 nach Italien zurück. In der Familie gibt es keine bekannten Suchtprobleme.

Mario K. besuchte die Schule in der Schweiz, absolvierte eine Lehre als Textilpfleger und war nachher als Versicherungsberater tätig. In seiner Jugend spielte er aktiv Fußball und verkehrte in einer Clique, in der häufig Cannabis geraucht wurde.
Nach der Trennung von seiner ersten Freundin begann er mit dreiundzwanzig Jahren, harte Drogen zu konsumieren. Später war er mit einer Frau verheiratet, die ebenfalls Drogenprobleme hatte. Als sie eine stationäre Therapie begann, trennte sie sich von ihm. Diese Trennung war für Mario K. äußerst schmerzlich und er stürzte in die Drogenszene ab. Er beging verschiedene Beschaffungsdelikte, weswegen er eine stationäre Maßnahme absolvieren musste, die er jedoch vorzeitig abbrach. Mario K. begann eine Methadonbehandlung mit psychotherapeutischer Begleitung. Allerdings konsumierte er nebenbei immer wieder Heroin. In dieser Zeit lernte er seine heutige Verlobte kennen, die ihrerseits drogensüchtig ist. Nach längerer Arbeitsunterbrechung wurde er im Januar 1998 in ein Beschäftigungsprogramm aufgenommen, in dem er bis zum Herbst 1999 halbtags arbeitete, dies ziemlich zuverlässig.

Mario K. begann im Sommer 1998, zusammen mit seiner damals schwangeren Verlobten, die Heroinbehandlung im Zokl2. Zu Beginn konsumierte er Heroin intravenös. Als im August 1998 der gemeinsame Sohn zur Welt kam, stieg er auf Herointabletten um. Dies ist im Verlauf einer Therapie ein großer Erfolg, da Herointabletten keine schnelle Anflutung der Droge im Gehirn - also keinen Flash - bewirken und Zeichen sind, dass sich die psychische Fixierung des Patienten auf die Droge gelockert hat. Neben der Heroinsubstitution behandelten wir ihn wegen generalisierten Ängsten und einer rezidivierenden depressiven Störung mit Antidepressiva. Zu den Gesprächen mit der therapeutischen Bezugsperson erschien er ziemlich regelmäßig, im Krankheitsfall meldete er sich im Allgemeinen ab. In den Gesprächen zeigte er sich kooperativ und offen. Seine Familie nahm einen zentralen Platz ein. Nachdem der neugeborene Sohn den Entzug im Spital hinter sich hatte, konnte das junge Paar das Kind nach Hause nehmen. Bald zeigten sich jedoch Überforderungssymptome - die junge Frau griff vermehrt zu Rohypnol, Mario K. verstrickte sich in Konflikte mit anderen Patient(inn)en, wobei er große Wut entwickeln konnte.

Im Frühjahr 1999 kam es zu einer deutlichen Destabilisierung der Situation - Mario K. erfuhr, dass er eine Haftstrafe antreten musste. Dies für ein Delikt, das ca. fünf Jahre zurückliegt. Alle Versuche, diese Haftstrafe in eine ambulante Maßnahme umzuwandeln, scheiterten. Mario K. war sehr enttäuscht und reagierte mit vermehrter Angst, bulimischen Attacken sowie ansteigendem Rohypnol- und Kokainkonsum. Auch in der Partnerschaft traten vermehrt Konflikte auf. Mario K. musste eine eigene Wohnung suchen, lebte aber trotzdem die meiste Zeit bei der Freundin. Das gemeinsame Kind kam in die Obhut eines Bruders der jungen Mutter.

Nachdem das Einsatzprogramm im Herbst 1999 beendet war, fiel seine Tagesstruktur weg. In den Wochen vor dem Haftantritt äußerte er mehrfach suizidale Absichten und gefährdete sich selbst mit hohen Dosen illegal konsumierter Medikamente. Immer wieder thematisierten wir mit dem Patienten die Einweisung in eine psychiatrische Klinik, was er jedoch ablehnte. Wiederholt äußerte er massive Ängste, seine Familie zu verlieren, wenn er ins Gefängnis müsse. Er trat die Haft nicht regulär an und musste von der Polizei zu Hause abgeholt werden. Dabei verletzte er sich selber derart, dass ihn der Polizeiarzt in eine psychiatrische Klinik überwies. Dort verbrachte er etwa zwei Wochen und wurde anschließend direkt 
in den Strafvollzug verlegt. Nach einigen Tagen erhielten wir die Nachricht, er habe einen weiteren Suizidversuch unternommen und sei in eine psychiatrische Klinik verlegt worden. Dies war die aktuelle Situation Ende 1999.

Nebst der Suchterkrankung liegt bei Mario K. eine Persönlichkeitsstörung vor. Bei Mario K. zeigte sich zwar zuerst eine Besserung seines Zustandes, bei Beginn der Behandlung änderten sich zudem seine Lebensumstände tiefgreifend - er lebte mit einer Frau zusammen, sie bekamen gemeinsam einen Sohn, den sie beide versorgten. Das bedeutet für alle Paare eine enorme psychische Belastung, drogenabhängige Eltern geraten dabei oft an den Rand der Überforderung. Die Behandlung wurde schwieriger, als weitere erschwerende Umstände auftraten (Haftbefehl), die bei Mario K. massive Ängste auslösten. Mario K. ist ein Beispiel dafür, dass manche Patient(inn)en ihre Lebenssituation zwar zeitweise stabilisieren können, sofern sie eine konstante Begleitung erhalten, aber immer wieder schwere Krisenphasen erleben. Die Behandlung solcher Patient(inn)en ist komplex, der Behandlungsverlauf meist sehr wechselhaft. Die Behandlung verfolgt langfristig haupsächlich das Ziel, die Lebenssituation zu stabilisieren. Weitergehende Integrationsschritte können meist erst nach einer längeren Behandlungszeit unternommen werden.

* Alle PatientInnendaten sind verändert. Ähnlichkeiten mit Ihnen allenfalls bekannten Personen sind zufällig.
ARUD Zürich

Konradstrasse 1

8005 Zürich

Schweiz

E-mail: arud@access.ch 